\title{
Überlegener Rückfallschutz auch im Alltag
}

Fragestellung: Wie ist die phasenprophylaktische Wirksamkeit von Lithium im Vergleich zu anderen Stimmungsstabilisierern unter realen Alltagsbedingungen?

Hintergrund: Randomisierte, kontrollierte Studien (RCT) sind der Goldstandard zur Untersuchung von Medikamenteneffekten. Die phasenprophylaktische Wirksamkeit von Lithium gegenüber Placebo konnte in den letzten Jahren durch neuere, methodisch hochwertige RCT nochmals bestätigt werden. Gegenüber anderen Pharmaka ist Lithium überlegen, da es als einziges Medikament in RCT ohne „angereichertes Design“ (enriched design) einen Schutz sowohl vor manischen als auch vor depressiven Phasen zeigte. Daher wird in der S3-Leitlinie Bipolare Störungen nur Lithium mit Empfehlungsgrad A zur Phasenprophylaxe empfohlen. In Deutschland ist auch allein Lithium uneingeschränkt zur Phasen-

Hayes JF, Marston L, Walters $\mathrm{K}$ et al. Lithium vs. valproate vs. olanzapine vs. quetiapine as maintenance monotherapy for bipolar disorder: a populationbased UK cohort study using electronic health records. World Psychiatry 2016; 15: $53-8$ prophylaxe zugelassen. Die Ergebnisse von RCT sind aber aufgrund einschränkender Faktoren nur bedingt auf die klinische Alltagssituation übertragbar. Vergleichsanalysen von Alltagsbehandlungen, möglichst in einer vollständigen, nicht selektierten
Patientenpopulation sind daher eine wichtige Ergänzung zu RCT.

Patienten und Methodik: Hayes et al. analysierten Daten von Patienten, die mit der Diagnose bipolar affektive Erkrankung zwischen 1995 und 2013 eine Monotherapie mit Lithium, Valproinsäure, Olanzapin oder Quetiapin begonnen hatten. Verglichen wurde, wie lange die Monotherapie beibehalten wurde. Die Monotherapie galt als gescheitert, wenn die Verordnung beendet oder durch ein zweites Psychopharmakon ergänzt wurde.

Ergebnisse: 1.505 Patienten mit Lithium-, 1.173 mit Valproinsäure-, 1.336 mit Olanzapin- und 1.075 mit Quetiapin-Monotherapie wurden analysiert. Mit Lithium behandelte Patienten verblieben signifikant länger auf der Monotherapie als die der anderen Gruppen. Die Zeit, nach der noch $25 \%$ der Patienten kontinuierlich die Monotherapie durchführten, betrug für Lithium durchschnittlich 2,05, für Olanzapin 1,13, für Valproinsäure 0,98 und für Quetiapin 0,76 Jahre. Die signifikante Überlegenheit von Lithium blieb auch nach statistischen Adjustierungen für Gruppenunterschiede bei den Basisparametern stabil.

Schlussfolgerung: Unter klinischen Alltagsbedingungen ist eine Phasenprophylaxe mit Lithium erfolgreicher als mit Valproinsäure, Olanzapin oder Quetiapin.

\section{- Kommentar von Tom Bschor, Berlin}

\section{Lithium ist das Phasenprophylaktikum der ersten Wahl}

Die Registerstudie zeigt eine phasenprophylaktische Überlegenheit von Lithium über die drei Vergleichssubstanzen, die auch allen statistischen Korrekturen standhielt. Allerdings sind die Raten einer erfolgreichen Monotherapie in allen Medikamentengruppen unbefriedigend. Selbst unter Lithium hatten nach zwei Jahren 75\% aller Patienten die Monotherapie beendet oder in eine Kombinationsbehandlung geändert. Verbesserungen in der phasenprophylaktischen Behandlung sind dringend erforderlich. RCT bieten als einziger Studientyp (Evidenzstufe I) den Vorteil, dass sich die verschiedenen Behandlungsgruppen gleichen und der einzige Unterschied in der Therapie besteht. Unterschiede im Ergebnis können daher auf die Intervention zurückgeführt werden. Jedoch stellen RCT eine künstliche Situation dar, sodass die Erkenntnisse nur eingeschränkt übertragen werden können. So führen strenge Einund Ausschlusskriterien zu nicht repräsentativen Studienpopulationen und umfangreiche Studienmaßnahmen unter anderem zu besserer Compliance, Studiendauer sowie Fallzahl sind begrenzt. Und RCT sind teuer und daher nur begrenzt durchführbar. Registerstudien haben diese Einschränkungen nicht, allerdings erfolgt die Zuteilung auf die verschiedenen Medikamente nicht per Zufall, sodass Unterschiede in den Ergebnissen auch auf unterschiedliche Patientencharakteristika zurück- geführt werden könnten (Evidenzstufe III). Durch statistische Korrekturen versucht man, diese Verzerrung zu minimieren. Beide Studientypen ergänzen sich ideal. Ein Therapieeffekt kann als abgesichert angesehen werden, wenn RCT und Registerstudien zu denselben Resultaten führen. Dies ist für die Phasenprophylaxe mit Lithium der Fall: Sie ist allen anderen in dieser Indikation eingesetzten Pharmaka überlegen. In Übereinstimmung mit der S3-Leitlinie Bipolare Störungen kann bestätigt werden, dass Lithium das Phasenprophylaktikum der ersten Wahl ist, das jedem bipolar affektiv erkrankten Patienten ohne eindeutige Lithiumkontraindikation vorrangig zur Phasenprophylaxe angeboten werden sollte.

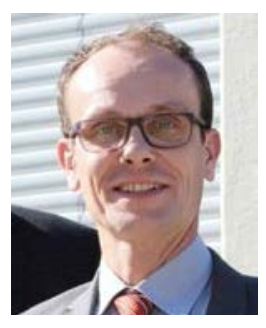

Prof. Dr. med. Tom Bschor, Berlin

Abteilung für Psychiatrie, Schlosspark-Klinik, Berlin E-Mail: bschor@schlosspark-klinik.de 